\section{AB1384 ARE THE SPANISH HOSPITALARIAN EMERGENCY UNITS PREPARED FOR THE DIAGNOSTIC AND THERAPEUTIC CARE OF URGENT RHEUMATOLOGICAL PATHOLOGY?}

C.A. Guillen-Astete ${ }^{1}$, I. Miguens-Blanco ${ }^{2}$, G. Rodriguez De Lema Tapetado ${ }^{3}$, J. Short-Apellaniz ${ }^{3} .{ }^{1}$ Rheumatology Department, Ramon y Cajal University Hospital; ${ }^{2}$ Gregorio Marañón University Hospital; ${ }^{3}$ Jimenez Díaz Foundation, Madrid, Spain

Background: The rheumatological emergencies are considered a pathology of low complexity by the classification and triage systems of the Emergency Departments (ED). However, their frequency ranks first in reasons for consultation. Although the majority of these reasons for consultation do not imply immediate urgency, some autoimmune diseases and their manifestations in the locomotor system are underdiagnosed, causing a delay in the referral to the specialist and the initiation of appropriate treatment.

Objectives: To determine the baseline knowledge level of the medical staff of the EDs in the diagnosis and treatment of rheumatological emergencies.

Methods: A survey was designed and distributed in 18 Spanish EDs. The choice of centres was made exclusively by availability and logistical access. In all cases, only medical personnel were surveyed. The survey was distributed through an electronic link to an online form created on the Google Forms platform. The first section of the questionnaire collected demographic data from the surveyed and the SU in which he performs his clinical activity. The second part compiled several aspects of general knowledge, training in diagnostic techniques and therapeutic behaviours.

Results: Of a potential 290 recipients, the survey was answered by 267 physicians. $68 \%$ of the surveyed were specialists in Family and Community Medicine, $25 \%$ in Internal Medicine and $7 \%$ were from other specialities. $25 \%$ declared having more than ten years of experience, $61 \%$ between 5 and ten years, and $14 \%$ less than five years. $91.7 \%$ of the people polled stated that the management of the non-traumatic pathology of the locomotor system was part of their usual work, while $8.3 \%$ indicated that this activity was within the responsibility of the Emergency Traumatologist. The proportion of surveyed who declared themselves capable of performing the following procedures were: knee arthrocentesis, $91.7 \%$; Shoulder infiltration, 75\%; Shoulder bursae puncture and fluid aspiration, $16.7 \%$; Joint ankle infiltration, 2.3\%; Infiltration in the carpal tunnel, 3.5\%; drainage of a patellar bursa, $14.3 \%$; drainage of an olecranon bursa, $12.1 \%$; Infiltrate the elbow, $21.2 \%$; Drain a popliteal cyst, $2.3 \%$. In the last three months, $38.5 \%$ of the polled said they had never obtained a joint or bursal fluid for diagnostic purposes, while $30.8 \%$ said they had done so only once. In the last three months, $61.5 \%$ of the assessed suspected once, giant cell arteritis. More than $60 \%$ of the surveyed felt confident of suspecting an outbreak of gout, rheumatoid arthritis, spondyloarthritis, rheumatic polymyalgia, giant cell arteritis, vertebral crushing and chondrocalcinosis based on the clinical signs. Less than $20 \%$ of the surveyed reported being able to suspect a connective tissue disorder or a vasculitis.

Conclusions: It is our understanding that the teaching priorities are in the field of training in diagnostic techniques, infiltrations other than those of large joints, clinical suspicion of autoimmune systemic diseases and diagnostic management of soft tissue pathology. Those topics must be included in further teaching initiatives to improve the quality of emergency units healthcare in the field of rheumatological urgencies.

Disclosure of Interest: None declared

DOI: 10.1136/annrheumdis-2018-eular.7131

\section{AB1385 \#RHEUMJC: 3 YEAR ANALYSIS OF A TWITTER BASED RHEUMATOLOGY JOURNAL CLUB}

C. Collins ${ }^{1}$, J. Campos ${ }^{2}$, A. Isabelle ${ }^{3}$, S. Bhana ${ }^{4}$, A. Jayatilleke ${ }^{5}$, P. Sufka ${ }^{6}$. ${ }^{1}$ Rheumatology, MedStar Washington Hospital Center, Washington, USA;

${ }^{2}$ Rheumatology, Puerta de Hierro Majadahonda University Hospital, Madrid, Spain; ${ }^{3}$ Rheumatology, National Jewish Health, Denver, ${ }^{4}$ Crystal Run Healthcare, Middletown; ${ }^{5}$ Rheumatology, Drexel University College of Medicine, Philadelphia; ${ }^{6}$ Rheumatology, HealthPartners, Saint Paul, USA

Background: Twitter is an increasingly popular platform for discussion and engagement amongst healthcare professionals. \#RheumJC is a Twitter-based international rheumatology journal club which has occurred approximately once a month for the past 3 years. Here we describe participant analysis and survey results from the past 36 months of this initiative.

Methods: A \#RheumJC development team, consisting of academic and private practice rheumatologists as well as rheumatology Fellows in Training (FIT), was created to help define the structure and moderate the online discussions. Based on results from an initial needs assessment survey, a structured journal club format was developed. A total of 23 different online journal clubs were conducted between January 2015 and December 2017, each consisting of both "live" one hour chats, as well as a full 24 hours to allow for asynchronous participation for each session. An analysis of the different sessions was performed to assess participant demographics and participation rates. Additionally, follow up surveys were conducted after the 4th, 10th and 23rd journal club sessions to assess metrics of satisfaction and identify additional strengths or barriers to participation.

Results: Over the 36 month observation period, a total of 646 individuals from 36 different countries tweeted with the hashtag \#RheumJC. Over $90 \%$ of these tweets occurred during the live chat sessions. While the majority of participants were rheumatologists, over 10 different medical fields were represented. The Twitter account @RheumJC currently has almost 2700 followers from 36 different countries, with the USA and UK representing the largest numbers of followers (36.4\% and $15.7 \%$ respectively), and $31.9 \%$ of all accounts originating from the European continent. The affiliated mailing list for \#RheumJC has over $235 \mathrm{sub}$ scribers. The most recent survey (done after the 23rd journal club session) recorded responses from 37 individuals from 13 different countries who indicated they had participated or followed along in at least one of the journal clubs. $74 \%$ of the respondents indicated they had participated in at least 2 or more different sessions. The majority $(86 \%)$ indicated they were either satisfied or very satisfied with the \#RheumJC initiative. $31 \%$ of respondents admitted that they had only observed some of the sessions and not joined in the actual discussion suggesting that the total number of \#RheumJC participants could be greater than the participation data presented here. Of interest, $9 \%$ of respondents indicated they had joined Twitter solely because of \#RheumJC, and another 29\% stated that \#RheumJC had increased their use of Twitter as a tool for medical education. The results from this survey closely mimic the responses from prior surveys suggesting an enduring satisfaction with the initiative.

Conclusions: \#RheumJC is a novel and popular approach to the traditional medical journal club, bringing together people from around the globe and across specialties to discuss current rheumatology literature utilising Twitter as a medium for medical education. Given its continued success for over 3 years now, the \#RheumJC initiative has proven itself a valuable addition to the rheumatology medical education community.

Disclosure of Interest: None declared

DOI: 10.1136/annrheumdis-2018-eular.3486

\section{AB1386 PHYSICIAN AWARENESS OF RHEUMATIC IMMUNE- RELATED ADVERSE EVENTS IN CANCER PATIENTS TREATED WITH IMMUNE CHECKPOINT INHIBITORS}

F. Araújo ${ }^{1,2}$, J.E. Fonseca ${ }^{3,4} \cdot{ }^{1}$ Rheumatology and Osteoporosis Unit, Hospital de Sant'Ana, SCML, Cascais; ${ }^{2}$ Institute of Microbiology, Faculdade de Medicina da Universidade de Lisboa; ${ }^{3}$ Rheumatology Research Unit, Instituto de Medicina Molecular, Faculdade de Medicina, Universidade de Lisboa, Portugal;

${ }^{4}$ Rheumatology Department, Hospital de Santa Maria, Lisbon Academic Medical Centre, Portugal., Lisbon, Portugal

Background: Immune checkpoint inhibitors (ICI) are novel and promising therapies for the treatment of a range of cancer types, acting through stimulation of the patient's immune system to engage on tumour cells. This enhanced immune sys tem may potentially cross-react against any organ system, and reporting of rheu matic immune-related adverse events (irAE) has been growing.

Objectives: To evaluate awareness of treatment with $\mathrm{ICI}$ and rheumatic irAE among Portuguese rheumatologists and oncologists.

Methods: A web-based questionnaire was sent in November 2017 to members of the Portuguese Society of Rheumatology and Portuguese Society of Oncology Aside from demographic variables, assessed domains included awareness and clinical experience with $\mathrm{ICI}$ and irAE, as well as educational needs on the topic and interest in participating in multidisciplinary approaches.

Results: Response rates were 61/221 (27.6\%) for rheumatologists and 13/653 $(2.0 \%)$ for oncologists. Demographics were similar in both groups, including mean age (39.9 and 41.1 years) and female gender (59\% and $53.8 \%$ ), respectively; the majority were consultant physicians $(67.2 \%$ and $69.2 \%)$ working at public hospitals $(86.9 \%$ and $92.3 \%$ ), respectively. Regarding $\mathrm{ICI}$, most rheumatologists had heard of but were unfamiliar $(63.9 \%)$ while most oncologists were at least moderately familiar $(92.3 \%)$ with such therapies. Almost all physicians were aware but more oncologists reported having patients with rheumatic irAE (46.2 vs $4.9 \%$ ); the most frequent were arthralgia and arthritis. These physicians were all moderately or very confident in managing these irAE. Most physicians considered that Rheumatology-Oncology multidisciplinary approaches would be of benefit and were interested in participating. Education on pathophysiology, epidemiology, clinical assessment and treatment was deemed necessary. Table 1 summarises the main results. 